\title{
Pyroresistivity in Conductive Polymer Composites: A Perspective on Recent Advances and New Applications
}

Yi Liu, ${ }^{1,2}$ Han Zhang, ${ }^{1,2}$ Harshit Porwal, ${ }^{1,2}$ James J.C. Busfield, ${ }^{1}$ Ton Peijs, ${ }^{1,2}$ and Emiliano Bilotti ${ }^{1,2, *}$

1. School of Engineering and Materials Science, Queen Mary University of London, Mile End Road, London E1 4NS, UK

2. Nanoforce Technology Ltd., Joseph Priestley Building, Queen Mary University of London, Mile End Road, E1 4NS London, UK

E-mail: e.bilotti@qmul.ac.uk

\section{Abstract}

Conductive polymer composites (CPCs) with pyroresistive behaviour is of interest for a widevariety of applications such as in new generations of safety batteries, resettable fuses, temperature sensors and self-regulating heating devices. Due to their ease of processing, low density, tuneable electrical properties, good oxidation resistance, and good flexibility and toughness, CPCs have become the preferable choice of pyroresistive materials in a number of applications. The pyroresistive behaviour of CPCs can be tuned to satisfy the specific requirements of different applications. In this perspective paper, recent progresses in the use of pyroresistive CPCs has been reviewed. In particular, various factors influencing their performances have been discussed and compared, in connection to the associated application, with a special focus on reproducibility and positive temperature coefficient (PTC) intensity levels. In addition, remaining challenges have been identified, together with future prospects in this evolving field.

Keywords: Conductive polymer composites (CPCs), nanocomposites, Positive temperature coefficient (PTC), Safe batteries, Smart heaters, Temperature sensors 


\section{Introduction}

Conductive polymer composites (CPCs) have gained extensive attention due to their functionality and smartness in a variety of technological application areas including electronic devices, conductive coatings, electromagnetic shielding, flexible displays and sensors. ${ }^{1-7}$ Although polymers are usually regarded as electrically insulating, electrical conductivity can be introduced by adding conductive particles, forming $\mathrm{CPCs} .{ }^{8} \mathrm{~A}$ great advantage of $\mathrm{CPCs}$ is that their electrical and mechanical properties can be tailored according to the specific application needs, by selecting different polymers and conductive fillers and even introducing sensitivity to a number of stimuli, such as strain, humidity, temperature, liquid, degradation and damage. ${ }^{9-17}$ Certain CPCs, in particular, can show a pyroresistive ("pyro" is the Greek word for "fire" or "heat") behaviour, with the electrical resistivity suddenly changing with temperature. Pyroresistivity in CPCs has been widely utilized in applications ranging from temperature sensors, batteries, fuses, to smart heating devices (Figure 1).

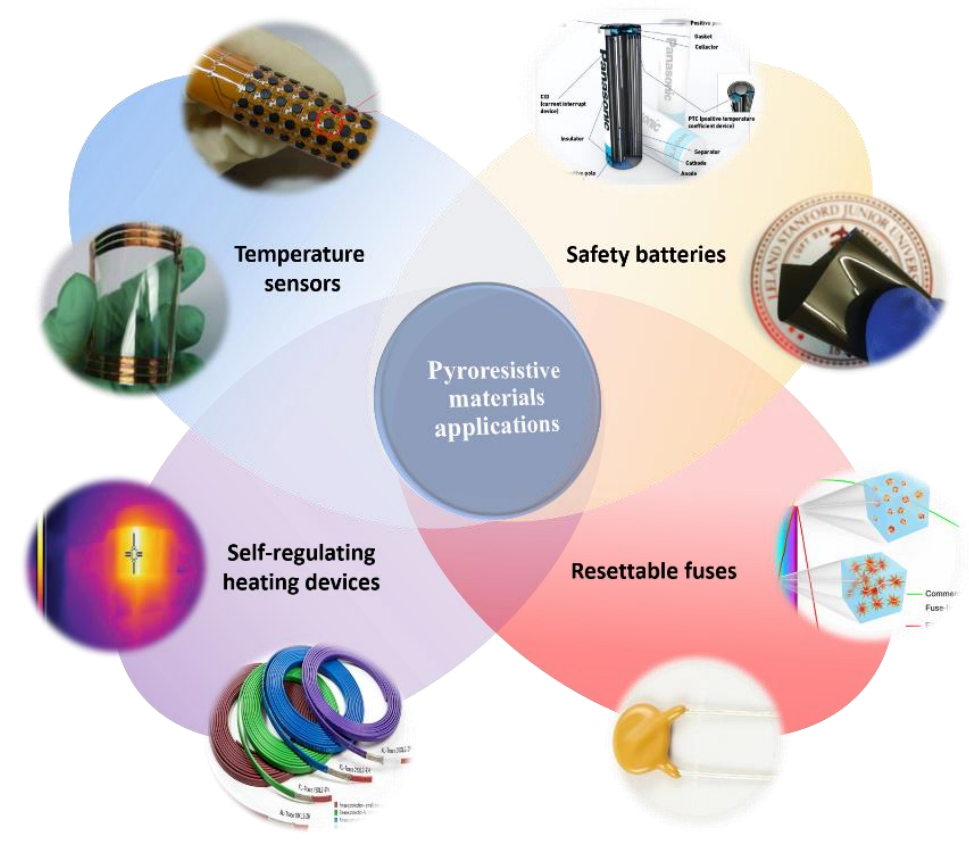

Figure 1. Applications of pyroresistive CPCs, ranging from temperature sensors, safety batteries, resettable fuses, to self-regulating heating devices. ${ }^{18-26}$ (Reproduced with permissions) 
Pyroresistivity can manifests itself in either an increase in electrical resistivity with increasing temperature - known as positive temperature coefficient (PTC) effect - or a decrease in electrical resistivity - known as negative temperature coefficient (NTC) effect. ${ }^{27,}{ }^{28}$ The PTC effect is generally believed to be associated with a mismatch of thermal expansion coefficient between polymer and filler, particularly evident in correspondence of a phase transition (e.g. melting) of the polymer matrix. ${ }^{29}$ This is due to the significant volume expansion at the transition, resulting in an increased inter-particle distance between the conductive fillers. ${ }^{30,} 31$ Conversely, when the composite cools down, the polymer contracts and the average interparticle distance decreases, reforming conductive pathways in the matrix and restoring electrical conductivity. It is worth noting that a NTC phenomenon is very often observed after the PTC phenomenon, with further increase in temperature.

In this perspective paper, recent progress in the field of pyroresistive CPCs has been briefly summarised, with a systematic discussion on the key aspects of pyroresistivity in CPCs, in connection with applications. Pyroresistive materials and devices with good repeatability, targeted PTC intensity, together with lightweight and controllable flexibility, are presented. This paper gives an overview of the governing factors, analyses recent applications based on pyroresistive materials, and discusses prospects for the future.

\section{Tuning positive pyroresistive behaviour}

Comparing with NTC effect, PTC effect has a wider field of applications in terms of safety protection. This is due to the increase in resistance when temperature raise, which limits both the current and temperature for safety reasons. 
Regardless of the final applications, four main aspects need to be considered for novel systems based on the PTC effect: (i) good repeatability; (ii) suitable PTC intensity; (iii) PTC switching temperature and; (iv) elimination of a NTC effect after PTC behaviour.

\section{- PTC repeatability}

Good repeatability is the basis for the long-term use of any PTC material. Therefore, great efforts have been made to design robust PTC materials with good repeatability. Unfortunately, this is not trivial to achieve. As an example, Jeon et al. found that in composites of polyethyle ne oxide (PEO) and micron-sized Ni particles, the resistivity could not recover to its original value after heating it to its melting point and cooling back to room temperature (RT). After six heating and cooling cycles, the resistivity of the single polymer system changed by more than one order of magnitude. ${ }^{21}$ Resistivity often increases with increasing thermal cycling, which makes the material impracticable for products with a long service life. ${ }^{32}$ The non-repeatability of resistivity was attributed to changes in the morphology of the filler network. By introducing polyethylene (PE) in the $\mathrm{PEO} / \mathrm{Ni}$ composite, the change in morphology of the $\mathrm{PEO} / \mathrm{Ni}$ phase could be reduced to a minimum as PE maintains its morphology during repeated thermal cycles. It has been shown that, over one hundred heating and cooling cycles, the resistivity of the $\mathrm{PEO} / \mathrm{PE} / \mathrm{Ni}$ composite did not show any distinct change in resistivity $(<0.5$ order of magnitude). This study showed that a binary polymer blend system can improve temperature repeatability in the potential use as temperature sensor towards applications such as medical diagnostics, food safety and environmental monitoring. ${ }^{21}$

Another strategy to enhance the stability or repeatability of PTC materials is through the addition of a secondary conductive filler. It has been widely accepted that the presence of carbon nanotubes (CNTs) is beneficial to increase repeatability and stability of nanocomposites, although often at the cost of sacrificing the PTC intensity. ${ }^{33}$ For example, the addition of a 
small amount of CNTs in carbon black (CB)/high density polyethylene (HDPE) composites could remarkably improve the electrical conductivity and thermal cyclic stability examined during the first three heating cycles as tested, in potential use of transistors, sensors and actuators. ${ }^{34}$ This was explained by the synergistic effect of the simultaneous presence of CNTs and $\mathrm{CB}$, which increases the number of conductive networks and improves stability ${ }^{35}$.

\section{- PTC intensity}

The PTC intensity, defined as the ratio of peak resistivity to the room temperature (RT) resistivity, is another key aspect when designing a smart pyroresistive material, since differe nt applications would potentially require different PTC intensity ranges. For example, in the area of electromagnetic interference (EMI) shielding or electrostatic discharge protection, the lowest possible PTC intensity is required to ensure good shielding properties. Whereas in the field of over-current protection or self-regulating heating, a high PTC intensity (ideally direct current cut-off) is required to ensure a rapid response of resistivity to temperature fluctuation. Therefore, it is of high importance to tune the PTC intensity in CPCs.

There are several ways to tune PTC intensity. One way is to change the conductive filler system. Experimental data suggests that CPCs with conductive fillers of larger average size and lower aspect ratio exhibit a higher PTC intensity and higher room temperature resistivity. ${ }^{36}$ Another way to tune the PTC intensity is to change the polymer matrix. It is suggested by many researchers that composites based on semi-crystalline polymer matrices, notably polyolefins, exhibit a higher PTC intensity than composites based on amorphous polymers. ${ }^{21,} 37$ The smaller change in resistivity in an amorphous polymer composite is related to the relatively small volume expansion, which is not sufficient to produce a significant and sudden change in resistivity. ${ }^{38,39}$ For example, composites with the same conductive filler type (Ni) and content, showed a PTC intensity of six to seven orders of magnitude when based on semi-crystalline 
polymers (i.e. PE, PEO, polyethylene adipate (PEA)), while only one to three orders of magnitude when based on amorphous polymers (i.e. polystyrene (PS), polyvinyl acetate (PVAc), polymethyl methacrylate (PMMA)). ${ }^{21}$

However, the correlation between PTC intensity and polymer crystallinity is not always a simple one as large PTC intensities have also been reported for amorphous CPCs. For example, Kar et al. reported a PTC intensity of five orders of magnitude for a PMMA composite containing 40 wt. $\%$ Ag coated glass beads. ${ }^{40}$ Here the PTC intensity could also be tuned by a physical-chemical treatment of the base polymer matrix. Those treatment of polymers including for instance, $\gamma$-ray irradiation, plasma treatment, and thermal treatment. He et al. demonstrated that $\gamma$-ray irradiation could modify the pyroresistive behaviour of an amorphous ethylene-propylene-diene terpolymer (EPDM)/CB composite, from showing only NTC behaviour to only PTC behaviour with a large PTC intensity (six orders of magnitude). ${ }^{41}$ This is assumed being resulted from the breakdown of agglomeration of $\mathrm{CB}$ aggregates by $\gamma$ irradiation-induced crosslinking of the EPDM matrices.

\section{- PTC switching temperature}

According to the switching mechanism mentioned above, the PTC phenomenon is triggered by a polymer phase transition, melting being the most evident. For semi-crystalline polymers, therefore, the critical switching temperature is typically close to the melting point of these polymers. For example, $\mathrm{HDPE} / \mathrm{CB}$ composites have a transition temperature around $130{ }^{\circ} \mathrm{C}$ while poly(vinylidene fluoride) (PVDF)/CB switch at around $170{ }^{\circ} \mathrm{C} .{ }^{37,42}$ For the amorphous polycarbonate $(\mathrm{PC})$, the switching temperature is close to its glass transition temperature $\left(\mathrm{T}_{\mathrm{g}}\right)$ of $150{ }^{\circ} \mathrm{C} .{ }^{43}$ But it is not uncommon to find exceptions to these observations in the scientific literature. Claims exist that the PTC switching temperature can also be tuned by additives and different filler loading. For instance, in a composite of PC and Ni coated graphite, the addition 
of polycaprolactone (PCL) helped to increase the thermal expansion mismatch between the polymers and fillers, resulting in a PTC effect well below the $\mathrm{Tg}_{\mathrm{g}}$ of the $\mathrm{PC} .{ }^{44}$ Moreover, it was found that with a change in Ag nanoparticle loading in a polymer blend comprising of PE, poly(butylene terephthalate) (PBT) and poly(m-xylene adipamide), the switching temperature could be tuned between 45 and $180{ }^{\circ} \mathrm{C} .{ }^{45}$ In general, the tuneability of the PTC switching temperature, by either changing the polymer matrix or using polymer blends, broadens the potential applications of PTC materials from fields like temperature sensors to self-regulating heaters.

\section{- NTC elimination}

The presence of an NTC effect constitutes a fundamental limitation to practical applications of PTC materials and devices, as it undermines the self-regulation mechanism, potentially resulting in severe safety issues. Several routes have been developed to eliminate the NTC effect, ranging from polymer blending methods, physical and chemical crosslinking methods, to the selection of specific conductive fillers or matrices. ${ }^{29,}{ }^{46}$ For instance, it has been reported that a CB filled ultra-high molecular weight polyethylene (UHMWPE)/polypropylene (PP) blend can exhibit a double PTC effect, which is due to the different melting temperatures of the two polymers, thus weakening the NTC effect. ${ }^{47}$ Similar two-step PTC effects have been achieved by Zhang et al. in HDPE/PVDF/carbon nanofibre (CNF) composites. ${ }^{48}$ The irradiation of the polymer matrices reduced filler movement in the melt phase thus lowering the NTC intensity. Moreover, the study of electro-beam irradiation by Seo et al. showed that in HDPE/CB composites, the NTC behaviour disappeared above a dosage of $60 \mathrm{kGy} .{ }^{49}$ The use of high viscosity matrices has also been reported to eliminate the NTC effect as the migration of nanofillers is strongly dependent on polymer viscosity. ${ }^{9}, 50$ 
Having briefly summarised the key aspects of pyroresistivity in CPCs, the following section will present novel applications where the pyroresistive effect is exploited. In particular, three main application areas will be discussed: (i) safety batteries; (ii) temperature sensors; and (iii) self-regulating heaters.

\section{Recent applications of pyroresistive materials}

\section{- Safety batteries}

Lithium-ion batteries (LIBs) are currently the most promising energy storage solution for electric vehicles, portable electric devices, and green grid energy storage. ${ }^{51}$ However, LIBs have been seriously plagued by safety issues such as high flammability of liquid electrolytes showing flash points (FPs) around room temperature (between 16 and $33{ }^{\circ} \mathrm{C}$ ), which has seriously hindered the application of LIBs. ${ }^{52}$ Therefore, several techniques and methods have been developed to enhance the safety of LIBs based on either external or internal protection mechanisms. ${ }^{53}$ External protection usually uses a control system, including temperature sensors, pressure valves and a variety of electronic devices, to protect and ensure normal operating conditions of LIBs. ${ }^{54}$ However, the occasional failures of external control systems, especially in exceptional conditions, cannot be completely ruled out. Hence, developing reliable internal protection mechanisms, combined with external protection systems, is particularly necessary for the safety of commercial LIBs.

Among the internal protecting components developed, few can be mentioned, including shutdown separators, PTC insert layers, as well as the use of flame retardant additives for liquid electrolyte or non-flammable solid-state electrolytes. ${ }^{55}$ It is well acknowledged that the employment of solid-state electrolytes can avoid the risk of using solvents, however, the 
performance of the battery is sacrificed due to their intrinsically low ionic conductivity compared to liquid electrolytes. ${ }^{56}$ For the separators, although they are effective in shutting down batteries, hence limiting operating voltages, they have the drawback of being irreversible, as the separators melt before thermal runaway. ${ }^{57,} 58$ Therefore, PTC electrodes seems to be an attractive safety strategy because of their high reliability, cost effectiveness, particularly easiness of use and reversibility. Herein, we will focus at reviewing the use of PTC materials in batteries, to achieve a safe and reversible transition, including a discussion of the underlying mechanis ms.

A typical LIB comprises a (flammable) organic electrolyte and two electrodes. Both electrodes are able to reversibly insert and remove $\mathrm{Li}^{+}$ions from their respective structures as shown in the schematic in Figure 2a. ${ }^{59}$ Under exceptional operating conditions, heat can build up quickly resulting in melting of the separator and internal shorting of the battery. This abnormal increase in temperature will cause thermal runaway and permanently damage the battery structure. To resolve this issue, researchers have developed PTC cathodes by incorporating a PTC compound into the active material layer. For example, Kise et al. made a PTC cathode via mixing the active material $\left(\mathrm{LiCoO}_{2}\right)$, with the PTC compound (HDPE/CB) and a binder. ${ }^{60}$ This worked in protecting the battery structure although the discharge capacity was reduced due to the high initial resistivity of the PTC cathodes. Moreover, this simple mixing method let to a sacrifice in PTC intensity by more than one order of magnitude. Also, the possibility that Lit ions intercalated and destroyed the CB network in the PTC compound could not be avoided. ${ }^{61}$ To overcome these limitations, multilayer structures were explored as an alternative PTC electrodes design strategy, by sandwiching a thin layer of PTC compound in between the electroactive $\mathrm{LiCoO}_{2}$ layer and current collector. For example, Bao et al. developed a PTC layer (called "thermoresponsive polymer switching" (TRPS) layer), based on a composite of PE and nanostructured spiky nickel particles coated with graphene ( $\mathrm{GrNi})$, and integrated it to 
the current collector (Figure $\mathbf{2 b}$ and c). ${ }^{18}$ During normal operating conditions, the conductive pathways in the PTC layer are connected, while above the switching temperature (abnormal conditions) of the PTC composite, the film instantaneously becomes electrically insulating and shuts down the battery. This safety switch allows a fast and reversible shut off of the LIB before battery damage or explosion, as demonstrated in Figure 2d, in which a sudden increase in temperature (induced externally by a heat gun) causes a connected LED to shut off. The LED lit up again with the removal of the external heating, which confirmed that there were no permanent structural damages to the battery nor degradation of battery performance. ${ }^{18}$

These research works have demonstrated the feasibility in battery protection by coating a pyroresistive layer on the current collector. Ideally, this safety layer should be made as thin as possible to reduce the distance between electrode and current collectors, especially when the coated PTC layer does not have high enough conductivity to favour electron extraction/injection. Therefore, there are still some challenges to tackle and room for further improvements.

(a)

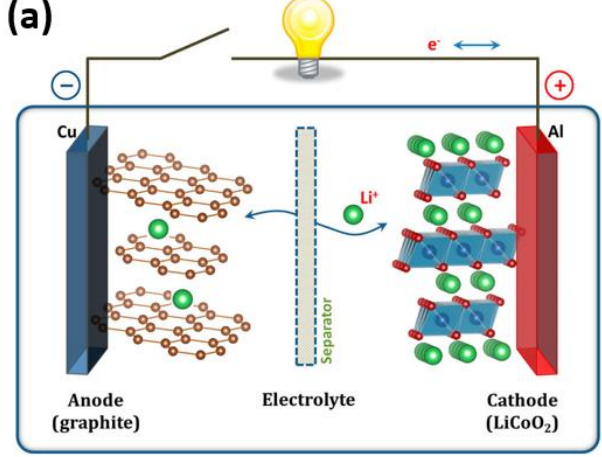

(d) (b)
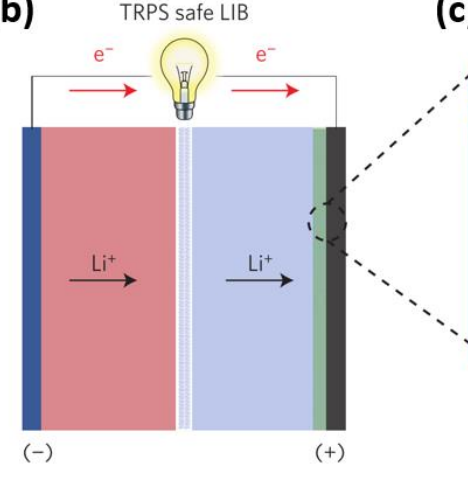

(c)

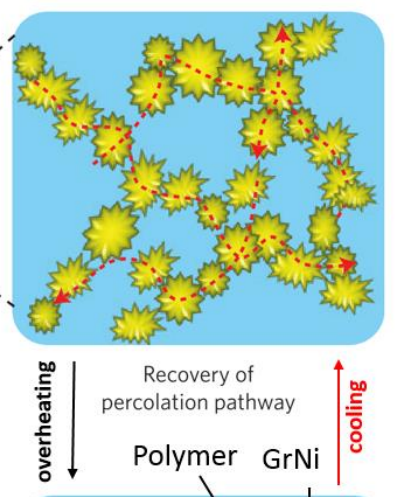

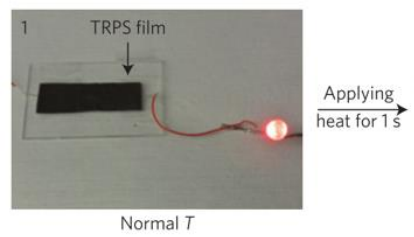
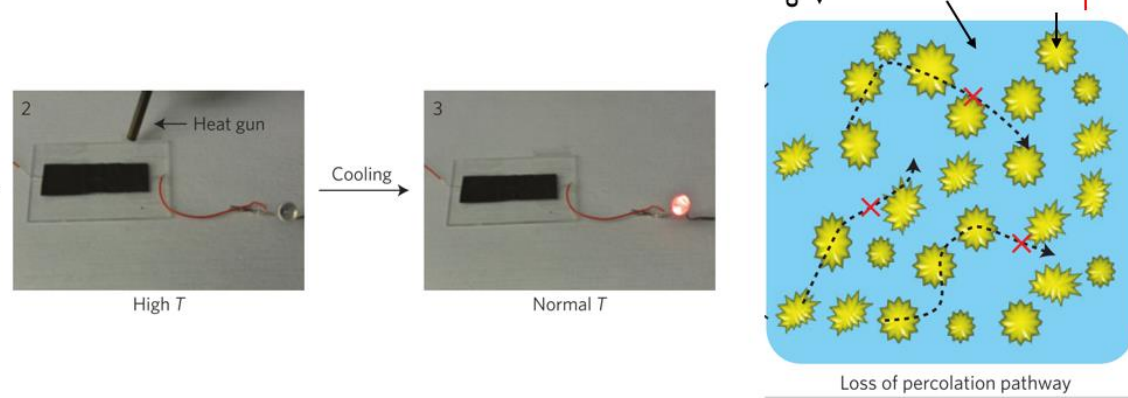
Figure 2. a) Schematic diagram of a typical LIB, consisting of an anode (e.g. graphite coated $\mathrm{Cu}$ ), a separator, a cathode (e.g. $\mathrm{LiCoO}_{2}$ coated $\mathrm{Al}$ ) and a flammable electrolyte; b) a thermoresponsive polymer switching (TRPS) layer based on nanostructured spiky Ni particles coated with graphene $(\mathrm{GrNi})$ as the filler embedded in a PE matrix and integrated into the current collector; c) switching mechanism of the TRPS material based on the breakdown of conductive pathways upon heating and recovery after cooling; d) demonstration of switching behaviour of a TRPS film using a LED as indicator and hot air gun to apply heat. ${ }^{18,59}$ (Reproduced with permission)

\section{- Thermistors}

PTC thermistors, such as temperature sensors and self-regulating heaters, are resistors with a positive pyroresistive effect. There are two types of PTC thermistors, one with a linear PTC character and one with a switching PTC character. The linear PTC type thermistors are usually used as temperature sensors with a large temperature range, while the switching PTC type thermistors are widely used in self-regulating heaters, resettable fuses, and temperature sensors at a specific temperature.

\section{- Temperature sensors}

The advantage of a linear type PTC temperature sensor is its wide temperature sensing range, while a switching type PTC temperature sensor can provide a better sensitivity. For example, polydimethylsiloxane (PDMS)/graphite composite was used to fabricate temperature sensor arrays on flexible polyimide films with a linear PTC character as shown in Figure $3 a^{22}$ The detectable temperature range could be from $40{ }^{\circ} \mathrm{C}$ to $110^{\circ} \mathrm{C}$, with $25 \mathrm{vol} \%$ of graphite loading. Issues like production in large scale, cost effectiveness and temperature accuracy of these composites are still to be overcome. Moreover, due to the relatively poor dispersion of the 
graphite, resistivity was low and hence the measured resistance of the composite increased with increasing bending radius of curvature.

For specific applications such as monitoring the human body temperature, a high temperature sensitivity is required. In this case, a switching type PTC thermistor is more desirable. Recently, a flexible wireless temperature sensor has been developed based on Ni micro-particle filled PEO/PE blends with a stable and adjustable PTC switching temperature as demonstrated in Figure 3b. In this case, Ni particles were preferentially located in the PEO phase of the polymer blend. By carefully selecting the molecular weights of the PEO, the PTC switching temperature could be tuned so that the sensor showed a strong PTC effect within the temperature range of 35 to $42{ }^{\circ} \mathrm{C}$, and accurate enough for human body temperature monitoring. A radio frequency identification (RFID) antenna was also integrated in the temperature sensor to enable wireless monitoring. ${ }^{21}$ Similarly, Yokota et al. developed an ultra-flexible temperature sensor, in the range of $29.8^{\circ} \mathrm{C}$ to $37.0^{\circ} \mathrm{C}$, based on semi-crystalline acrylate polymers and graphite, printed through stencil masks (Figure 3c). The device exhibited large resistance changes near body temperature with high repeatability (tested over 1800 thermal cycles) and a fast response time. ${ }^{62}$ This method can produce a flexible temperature sensor sheet, which also allows for large-area temperature mapping as shown in Figure 3d and e. When the temperature increases from $30{ }^{\circ} \mathrm{C}$ to $34.5{ }^{\circ} \mathrm{C}$, the current shows a large decrease, from $1 \mathrm{~mA}$ to $10 \mathrm{nA}$. This demonstrates the possibility of very precise and large area temperature monitoring.

Apart from human body temperature sensors, relatively high temperature sensor applications with PTC switching features are also in high demand. Therefore, various efforts have focussed on developing temperature sensors with switching temperatures exceeding $100{ }^{\circ} \mathrm{C}$. For example, Zha et al. recently used modified CB and MWCNT filled HDPE to fabricate high performance PTC composites as flexible temperature resistivity sensors with a switching temperature of around $130{ }^{\circ} \mathrm{C}$. Besides, the addition of small amounts of CNTs $(0.7 \mathrm{wt} . \%)$ was 
able to reduce the overall filler concentration but, surprisingly, provide a larger PTC intensity and improved repeatability. ${ }^{35}$

(a)

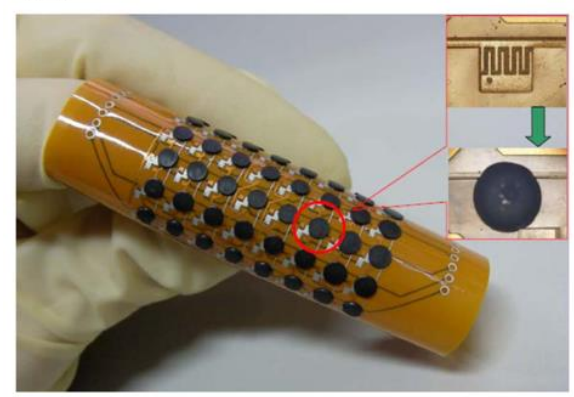

(d)

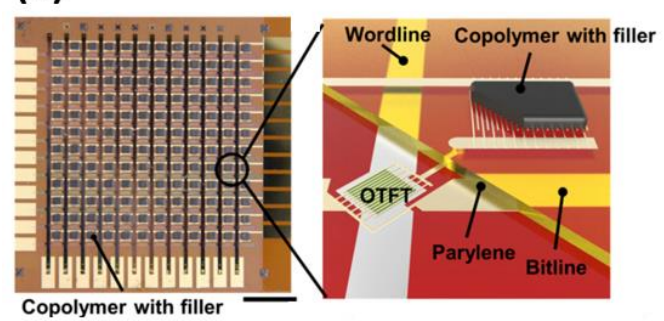

(b)

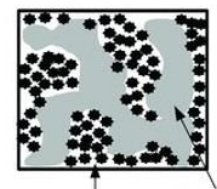

PEO with Ni Microparticles

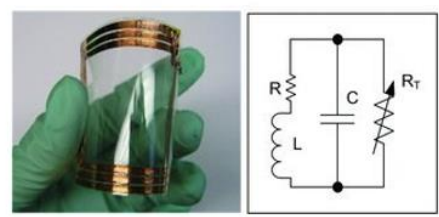

(e)

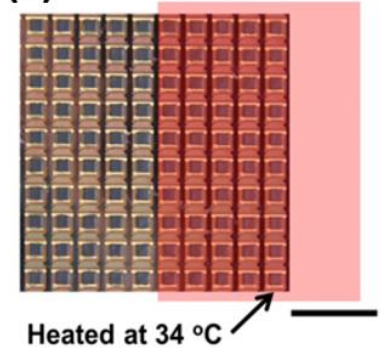

(c) $10^{\circ}$
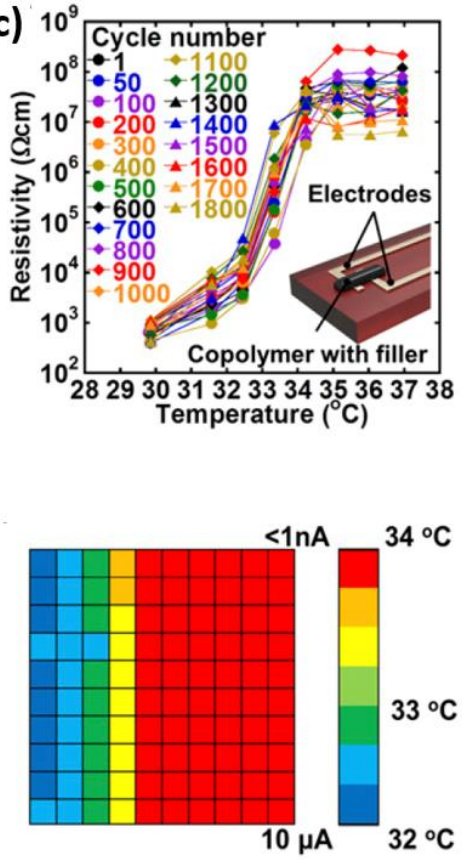

Figure 3. a) Temperature sensor arrays on flexible polyimide films with a linear PTC characteristic; b) a flexible wireless temperature sensor based on $\mathrm{Ni}$ micro-particle filled PEO/PE blends with a stable and adjustable PTC switching temperature; c) heat cycling tests for this temperature sensor up to 1800 cycles; d) flexible temperature sensor sheet (scale bar, $1 \mathrm{~cm}$.) with enlarged cross-sectional illustration of the matrix; e) area of the temperature sensor sheet heated to $34{ }^{\circ} \mathrm{C}$ (scale bar: $1 \mathrm{~cm}$ ) and temperature gradient mapping. ${ }^{21,22,62}$ (Reproduced with permissions).

\section{-Self-regulating heaters}

Another intrinsic nature of thermistors, that has been widely explored when making heating devices, is the Joule heating effect, also known as resistive heating. When a voltage is applied to a thermistor to generate sufficient current flowing through it, the heat generated results in a temperature increase. By combining the PTC effect with the Joule heating effect, a self- 
regulating heating device can be created which possesses an integrated safety temperature control system to avoid overheating. As the temperature of the heating device reached the PTC switching temperature, the resistance will increase dramatically, resulting in a reduction in current flow. Hence, heating through Joule heating is used to allow devices to maintain their switching temperature. For instance, Figure 4a shows a Joule heating specimen (PDMS/CB composite) reaching a temperature of $45^{\circ} \mathrm{C}$ (switching temperature) with an obvious increme nt in electrical resistance. Even with further increased voltage applied to promote the Joule heating effect, no further increment in temperature was observed as not enough current can pass through above the PTC temperature. ${ }^{19}$

However, most polymeric PTC heaters consisting of spherical fillers cannot reach high temperature range. ${ }^{19}$ Moreover, polymers used in traditional self-regulating compounds (e.g. HDPE/nanocarbon), particularly with a high filler content and high cross-link density, show typically a rather brittle mechanical behaviour which is not suitable for flexible and even wearable heating devices or heating mats. One way to tune the flexibility and PTC effect of a heating device while preserving the Joule heating properties is by adding a secondary thermoplastic elastomeric (TPE) phase to a HDPE/graphene nanoplatelet (GNP) composite. The selection of the TPE phase will greatly affect the morphology of the resulting blends, leading to an immiscible binary blend with either a fine or course droplet morphology, or a cocontinuous morphology. ${ }^{63}$ In the case of the HDPE/TPE/GNP system the temperature stabilised at the self-regulating temperature of around $120{ }^{\circ} \mathrm{C}$, with the thermal image showing a uniformly heated sample (Figure 4b). Developed materials' flexibility was greatly enhanced in these TPE modified composites as the blending radii could be reduced to $3 \mathrm{~mm} .{ }^{63}$

Our group recently presented a versatile sandwich-based design approach by connecting different CPCs with distinct pyro-resistive behaviours in series, achieving for the first time a high PTC intensity combined with excellent Joule heating as well as mechanical flexibility 
(Figure $4 \mathbf{c}$ and d). ${ }^{20}$ Here, the Ag coated glass spheres filled TPU composite acted as the switching unit, while a TPU/CNT composite at both ends of the sandwich device acted as the heating units. The use in this case of TPU as the polymer matrix, combined with the series configuration and the fillers selected, significantly improved the flexibility of such a heating device as shown in Figure 4c.

(a)

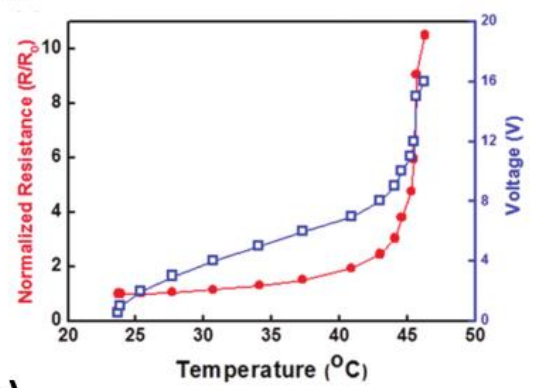

(c)

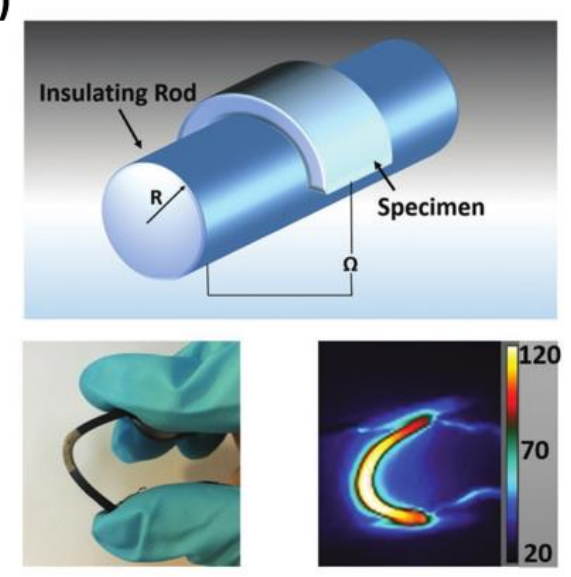

(b)
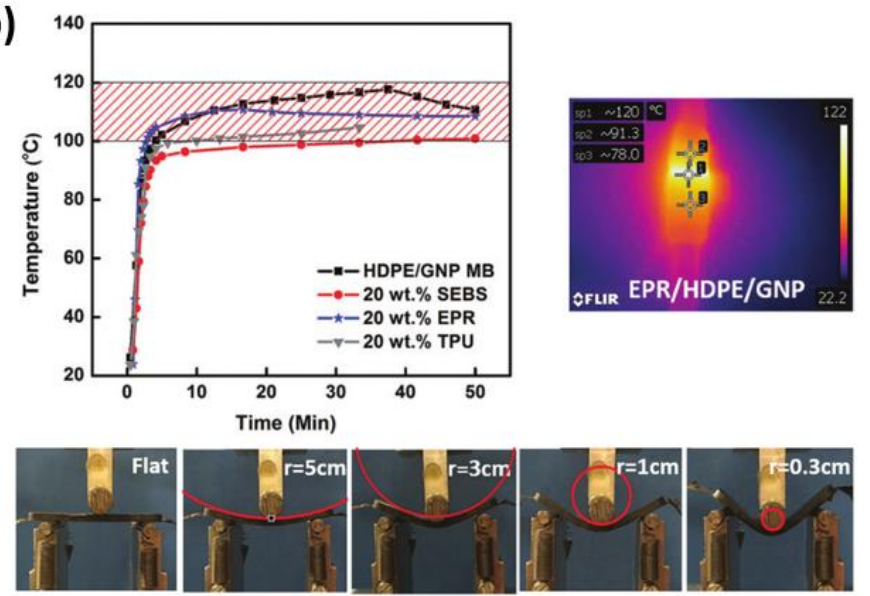

(d)

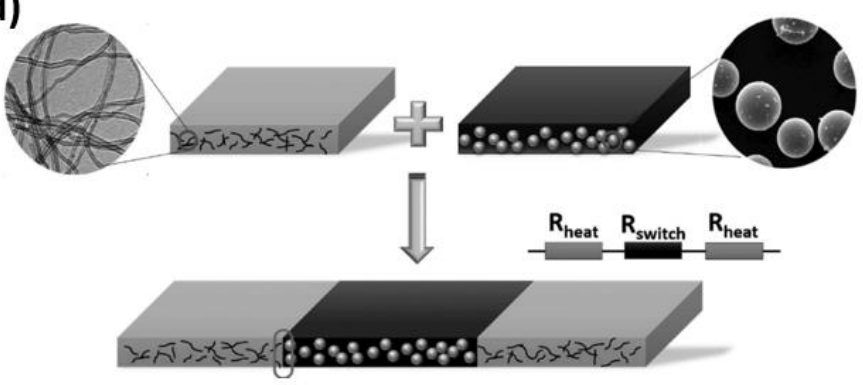

Figure 4. a) Resistance increases dramatically in PDMS/CB composites, allowing less current to flow; b) secondary thermoplastic elastomeric (TPE) phases filled HDPE/GNP composites with the temperature stabilised at the self-regulating temperature around $120^{\circ} \mathrm{C}$, and enhanced flexibility; c) and d) a simple and versatile sandwhich-based approach that tackles typical design compromises in smart self-regulating heating devices by connecting different CPCs in series with distinct pyro-resistive behaviours. The Ag coated glass spheres filled TPU composite acting as the switching unit, and TPU/CNT composites at both ends behaving as the 
heating units, leading to heating devices with significantly improved flexibility with preserved heating properties. ${ }^{19,20,63}$ (Reproduced with permission)

\section{Conclusions and Future Perspectives}

Pyroresistivity in CPC can be exploited for a wide range of practical applications. After having reviewed recent advances in understanding the main factors influencing the PTC effect (e.g. PTC repeatability, PTC intensity, PTC switching temperature and NTC elimination after PTC), we have focused on some of the most promising commercial applications: in safety batteries, as temperature sensors, and self-regulating heaters.

Great potential can be seen in using pyroresistive materials in novel batteries, to prevent thermal runaway and consequent explosion. However, the development of reliable materials for electrodes, with enhanced electrical conductivity, and in the future, flexibility and stretchability, are still far to be fully achieved. More efforts are required toward high performance thin PTC films, with large PTC intensity, stability, at the same time, high electrical conductivity.

For temperature sensors and self-regulating heaters, accuracy and cyclic reliability are the key aspects that need to be tackled. Also in this case the addition of properties like mechanical flexibility and stretchability, would further broaden the potential applications. Two methodologies can be used to improve flexibility and stretchability. One is through novel device designs, such as the series assembly design mentioned above and the arts of origami and kirigami design with high reconfigurability and multiple functionality. ${ }^{64}$ The other is through exploring intrinsically flexible or stretchable materials. 
An additional desirable feature, to incorporate into pyroresistive materials, would be the possibility to do intelligent and remote control.

Gazing into the future, the field of pyroresistive materials is an exciting one and fairly openended, providing researchers to explore novel and interesting materials and design concepts. Many of these novel intelligent devices are expected to enter our daily lives in the near future.

\section{Acknowledgements}

The authors gratefully acknowledge the financial support from LMK Thermosafe Ltd., and Innovate UK (KTP number: KTP009619).

\section{Reference}

1

3

4

5

6

7

8

9 E. Bilotti, R. Zhang, H. Deng, M. Baxendale and T. Peijs, Journal of Materials Chemistry 20:94499455 (2010).

10 R. Zhang, H. Deng, R. Valenca, J. Jin, Q. Fu, E. Bilotti and T. Peijs, Composites Science and Technology 74:1-5 (2013).

11 T. Villmow, S. Pegel, A. John, R. Rentenberger and P. Pötschke, Materials Today 14:340-345 (2011).

12 H. Zhang, Y. Liu, M. Kuwata, E. Bilotti and T. Peijs, Composites Part A: Applied Science and Manufacturing 70:102-110 (2015).

13 H. Zhang, E. Bilotti and T. Peijs, Nanocomposites 1:167-184 (2015). 

212:2239-2245 (2015).

F. Mai, Y. Habibi, J.-M. Raquez, P. Dubois, J.-F. Feller, T. Peijs and E. Bilotti, Polymer 54:68186823 (2013).

J.-F. Feller, 6.10 Electrically Conductive Nanocomposites, in Comprehensive Composite Materials II. Elsevier, Oxford, pp. 248-314 (2018). and Technology 167:24-31 (2018). Bao, Nature Energy 1:15009 (2016). Advanced Functional Materials 27:1702253 (2017).

21 J. Jeon, H. B. R. Lee and Z. Bao, Advanced Materials 25:850-855 (2013).

22 W.-P. Shih, L.-C. Tsao, C.-W. Lee, M.-Y. Cheng, C. Chang, Y.-J. Yang and K.-C. Fan, Sensors 10:3597-3610 (2010). 11:7710-7718 (2017).

Tesla Model S - 18650 Cell Available from: http://www.invetr.com/control-andelectronics/ev-battery.

25 Raychem 10XL2-ZH Self Regulating Trace Heating Cable Available from: https://www.traceheatinguk.co.uk/raychem-10xl2-zh-self-regulating-trace-heating-cable$10 \mathrm{w} / \mathrm{m}$-at-5-c.

26 Y. Shi, H. Ha, A. Al - Sudani, C. J. Ellison and G. Yu, Advanced Materials 28:7921-7928 (2016).

27 K. Ohe and Y. Naito, Japanese Journal of Applied Physics 10:99 (1971).

28 Z. D. Xiang, T. Chen, Z. M. Li and X. C. Bian, Macromolecular Materials and Engineering 294:9195 (2009).

29 H. Xu, Y. Wu, D. Yang, J. Wang and H. Xie, Rev Adv Mater Sci 27:173-183 (2011).

30 J. Meyer, Polymer Engineering \& Science 13:462-468 (1973).

31 K. Matsushige, K. Kobayashi, N. Iwami, T. Horiuchi, E. Shitamori and M. Itoi, Thin Solid Films 273:128-131 (1996).

32 J. Meyer, Polymer Engineering \& Science 14:706-716 (1974).

33 E. Asare, A. Basir, W. Tu, H. Porwal, H. Zhang, Y. Liu, J. Evans, M. Newton, T. Peijs and E. Bilotti, Nanocomposites 2:58-64 (2016).

34 J. H. Lee, S. K. Kim and N. H. Kim, Scripta Materialia 55:1119-1122 (2006).

35 J.-W. Zha, D.-H. Wu, Y. Yang, Y.-H. Wu, R. K. Li and Z.-M. Dang, RSCAdvances 7:11338-11344 (2017).

36 E. Asare, J. Evans, M. Newton, T. Peijs and E. Bilotti, Materials \& Design 97:459-463 (2016).

37 Y. Luo, G. Wang, B. Zhang and Z. Zhang, European polymerjourna/34:1221-1227 (1998).

38 D. Pfefferkorn, S. Sonntag, S. O. Kyeremateng, Z. Funke, H. W. Kammer and J. Kres sler, Joumal of Polymer Science Part B: Polymer Physics 48:1893-1900 (2010).

39 C. Xiong, Z. Zhou, W. Xu, H. Hu, Y. Zhang and L. Dong, Carbon 43:1788-1792 (2005).

40 P. Kar and B. B. Khatua, Polymer Engineering \& Science 51:1780-1790 (2011).

41 X. He, L. Wang and X. Chen, Journal of Applied Polymer Science 80:1571-1574 (2001).

42 A. Kono, K. Shimizu, H. Nakano, Y. Goto, Y. Kobayashi, T. Ougizawa and H. Horibe, Polymer 53:1760-1764 (2012).

43 K. Zribi, J. F. Feller, K. Elleuch, A. Bourmaud and B. Elleuch, Polymers for Advanced Technologies 17:727-731 (2006).

44 P. Kar and B. Khatua, Polymer Composites 32:747-755 (2011).

45 A. Rybak, G. Boiteux, F. Melis and G. Seytre, Composites Science and Technology 70:410-416 (2010). 
$46 \quad$ H. Deng and Q. Fu, Macromolecular Rapid Communications 38:(2017).

47 J. Feng and C.-M. Chan, Polymer 41:4559-4565 (2000).

48 X. Zhang, S. Zheng, H. Zou, X. Zheng, Z. Liu, W. Yang and M. Yang, Composites Part A: Applied Science and Manufacturing 94:21-31 (2017).

49 M.-K. Seo, K.-Y. Rhee and S.-J. Park, Current Applied Physics 11:428-433 (2011).

50 Y. Xi, H. Ishikawa, Y. Bin and M. Matsuo, Carbon 42:1699-1706 (2004).

51 Y. Shi, L. Peng, Y. Ding, Y. Zhao and G. Yu, Chemical Society Reviews 44:6684-6696 (2015).

52 J. Wen, Y. Yu and C. Chen, Materials express 2:197-212 (2012).

53 W. Ji, F. Wang, D. Liu, J. Qian, Y. Cao, Z. Chen, H. Yang and X. Ai, Journal of Materials Chemistry A 4:11239-11246 (2016).

54 L. Lu, X. Han, J. Li, J. Hua and M. Ouyang, Journal of Power Sources 226:272-288 (2013).

55 P. Balakrishnan, R. Ramesh and T. P. Kumar, Journal of Power Sources 155:401-414 (2006).

56 D. H. Wong, J. L. Thelen, Y. Fu, D. Devaux, A. A. Pandya, V. S. Battaglia, N. P. Balsara and J. M. DeSimone, Proceedings of the National Academy of Sciences 111:3327-3331 (2014).

57 S. S. Zhang, Journal of Power Sources 164:351-364 (2007).

58 H. Xiang, H. Xu, Z. Wang and C. Chen, Journal of Power Sources 173:562-564 (2007).

59 J. B. Goodenough and K.-S. Park, Journal of the American Chemical Society 135:1167-1176 (2013).

60 M. Kise, S. Yoshioka, K. Hamano, D. Takemura, T. Nishimura, H. Urushibata and H. Yoshiyasu, Journal of power sources 146:775-778 (2005).

61 X. M. Feng, X.P. Ai and H. X. Yang, Electrochemistry Communications 6:1021-1024 (2004).

62 T. Yokota, Y. Inoue, Y. Terakawa, J. Reeder, M. Kaltenbrunner, T. Ware, K. Yang, K. Mabuchi, T. Murakawa and M. Sekino, Proceedings of the National Academy of Sciences 112:14533-14538 (2015)

63 Y. Liu, H. Zhang, H. Porwal, W. Tu, K. Wan, J. Evans, M. Newton, J. J. C. Busfield, T. Peijs and E. Bilotti, Journal of Materials Chemistry C 6:2760-2768 (2018).

64 L. Xu, T. C. Shyu and N. A. Kotov, ACS nano 11:7587-7599 (2017). 